\title{
Energy Efficiency Optimization for multiuser OFDM-based Cognitive Heterogeneous networks
}

\author{
Bing Ning ${ }^{1 *}$, Aihua Zhang ${ }^{1}$, Wanming $\mathrm{Hao}^{2}$, Jianjun $\mathrm{Li}^{1}$ and Shouyi Yang ${ }^{3}$ \\ ${ }^{1}$ School of Electronic and Information Engineering, Zhongyuan University of Technology, \\ Zhengzhou, 450007-China \\ ${ }^{2} 5 \mathrm{G}$ Innovation Centre, Institute of Communication Systems, University of Surrey, Guildford GU2 7XH, \\ U.K. \\ ${ }^{3}$ School of Information Engineering, Zhengzhou University, \\ Zhengzhou, 450001-China \\ [E-mail: ningbing0106@gmail.com] \\ *Corresponding author: Bing Ning
}

Received August 2, 2018; revised October 24, 2018; accepted November 5, 2018; published June 30, 2019

\begin{abstract}
Reducing the interference to the licensed mobile users and obtaining the energy efficiency are key issues in cognitive heterogeneous networks. A corresponding rate loss constraint is proposed to be used for the sensing-based spectrum sharing (SBSS) model in cognitive heterogeneous networks in this paper. Resource allocation optimization strategy is designed for the maximum energy efficiency under the proposed interference constraint together with average transmission power constraint. An efficiency algorithm is studied to maximize energy efficiency due to the nonconvex optimal problem. Furthermore, the relationship between the proposed protection criterion and the conventional interference constraint strategy under imperfect sensing condition for the SBSS model is also investigated, and we found that the conventional interference threshold can be regarded as the upper bound of the maximum rate loss that the primary user could tolerate. Simulation results have shown the effectiveness of the proposed protection criterion overcome the conventional interference power constraint.
\end{abstract}

Keywords: Cognitive Heterogeneous Network, OFDM, spectrum sensing, resource allocation, energy efficiency.

This study was funded by the National Natural Science Foundation of China (Grant no. 61501530) , Henan Educational Committee (Grant no. 16A510012), and Supported by Foundation for University Key Teachers of Henan Province. 


\section{Introduction}

As multimedia data transmission becoming the mainstream, the mobile data traffic has increased dramatically in the wireless communication network, which requires greater spectrum resources to meet the growing demand for wireless communication applications. However, the recent reports by Federal Communications Commission (FCC) have shown that there is a persistent problem of low spectrum utilization in the licensed frequency bands. Cognitive radio (CR) [1] is an important technology for efficiently increasing the utilization of available radio spectrum. However, in order to meet the requirement of the multimedia data transmission, cognitive radio network is expected to be heterogeneous with different spectrum bands, heterogeneous cell structures and various services, that is named heterogeneous CR network. In heterogeneous CR network where the licensed bands are assigned to primary users (PU) and secondary users (SU) simultaneously in different cognitive radio networks, different SUs equipped with multiple radio access technologies can flexibly access the "available" spectrum, when the interference to PU could be tolerable. In general, the operation for heterogeneous $\mathrm{CR}$ systems has three types: i) opportunistic spectrum access (OSA) [2], where the secondary user can access the licensed bands that only detected as idle; ii) spectrum sharing (SS) [3], where the secondary user is allowed to share the licensed bands only when the quality of servise (QoS) of PU is protected; iii) sensing-based spectrum sharing (SBSS) [4] [5], where the secondary user chooses the appropriate transmit power according to the sensing results for PU. Due to the higher spectrum efficiency in the SBSS model, this paper will focus on the study in this model.

Interference to the primary user has been regarded as one of the most challenging problems in heterogeneous CR networks and extensively investigated recently [6]-[13]. In [6], a common method that constrain the interference power during the SU transmission is proposed to protect the QoS of PUs. In [9], the tradeoff between interference alignment and the sum rate of SU is investigated in the multi-user multi-antenna CR networks. In [10], the nonstationary minimum-variance distortionless response beamformer is designed to perform the fast speed of convergence and solve the problem of interference to PU. The conventional interference power constraint by reducing the transmission power of SU has been always used to protect the interests of PU in many researches. In [11], a general multi-ring model in the heterogeneous CR network is proposed to investigate the relationship between the SUs' distribution and the interference to PU. The interference generated from heterogeneous CR network is investigated by employing several strategies, such as the SU's transmission power control, the secondary transmitters competitive strategy and the secondary receivers association scheme. It is well known that there are many factors that could cause the interference to PU, such as the secondary user deployment density and the channel characteristics between the secondary user and the primary user. However, it is unreasonable only from the SU's perspective to minimize the interference to PU by the traditional interference power constraint or the distribution density of SU. In [13], the author proposed the rate loss constraint (RLC) for spectrum sharing model in CR networks. The new criterion is more directly related to the PU transmission and obtains improved capacity over the conventional criterion, compared to the conventional interference power constraint. However, the energy efficiency and adaptive power allocation was not considered in the obove-mentioned papers. 
With the tremendous increasing requirement of multimedia data transmission and energy saving, energy efficiency (EE) has become the key issue for CR networks in recent researches [14]-[18]. In [14], energy consumption and access strategy is considered in both half-duplex and full-duplex CR network. In [15], the problem of numerous SUs accessing the licensed spectrum is considered. Energy efficiency problem under the communication rate constraint and transmission power constraint to maximize the feasible accessing number of SU is studied. In [16], the tradeoff between the secrecy throughput and the energy consumption with different spectrum access schemes is investigated in CR networks. The above studies performed the energy efficiency optimization problem based on the perfect channel state information (CSI) without considering the status of PU and only considered the single architecture in CR network. However, investigating the maximum energy efficiency problem with imperfect status information of PU for heterogeneous CR networks is meaningful.

In this paper, we design the resource allocation optimization strategy include sensing time and power allocation to obtain the maximum energy efficiency for the sensing-based spectrum sharing model in multi-band OFDM cognitive heterogeneous network that employs simultaneous multiband detection. A sensing-based rate loss constraint (SRLC) under imperfect spectrum sensing as the new criterion in cognitive heterogeneous networks is proposed. An efficiency algorithm is studied to solve the nonconvex optimal problem of energy efficiency. Furthermore, the relationship between the proposed protection criterion and the conventional interference constraint is studied. Numerical simulation results have proved that the effectiveness of the proposed interference strategy is overcome the conventional interference temperature strategy.

The main contributions of this study are summarized as follows:

1) The sensing-based rate loss constraint based on the different sensing results for cognitive heterogeneous networks is proposed to protect the PUs and constrain SU, which makes it suitable for the sensing-based spectrum sharing model in cognitive heterogeneous networks, while [13] only considered SS model without sensing.

2) The energy efficiency resource optimization problem for cognitive heterogeneous networks is studied under these two different schemes, (i) the SRLC and the transmission power constraint; (ii) the conventional interference and transmission power constraint. Considering the nonconvex optimization problem, this paper transforms the nonlinear stochastic fractional energy efficiency problem into the equivalent nonlinear parametric programming through theoretical demonstration.

3) The relationship between the CRLC and the conventional interference power constraint under the imperfect spectrum sensing for the SBSS model is also investigated through theoretical demonstration, and we conclude that the interference power constraint threshold can be regarded as an upper bound of the maximum rate loss that the primary user could tolerate.

The rest of this paper is organized as follows. The system model for multi-band OFDM cognitive heterogeneous network is introduced in Section 2. Section 3 and 4 present the energy efficiency resource allocation optimization strategies under two above-mentioned schemes. Section 5 studies the relationship between the CRLC and the conventional interference power constraint for the sensing-based spectrum sharing model. Section 6 validates the effectiveness of the proposed strategy compared to the conditional strategy by numerical simulation results. Section 7 concludes the paper. 


\section{System Model}

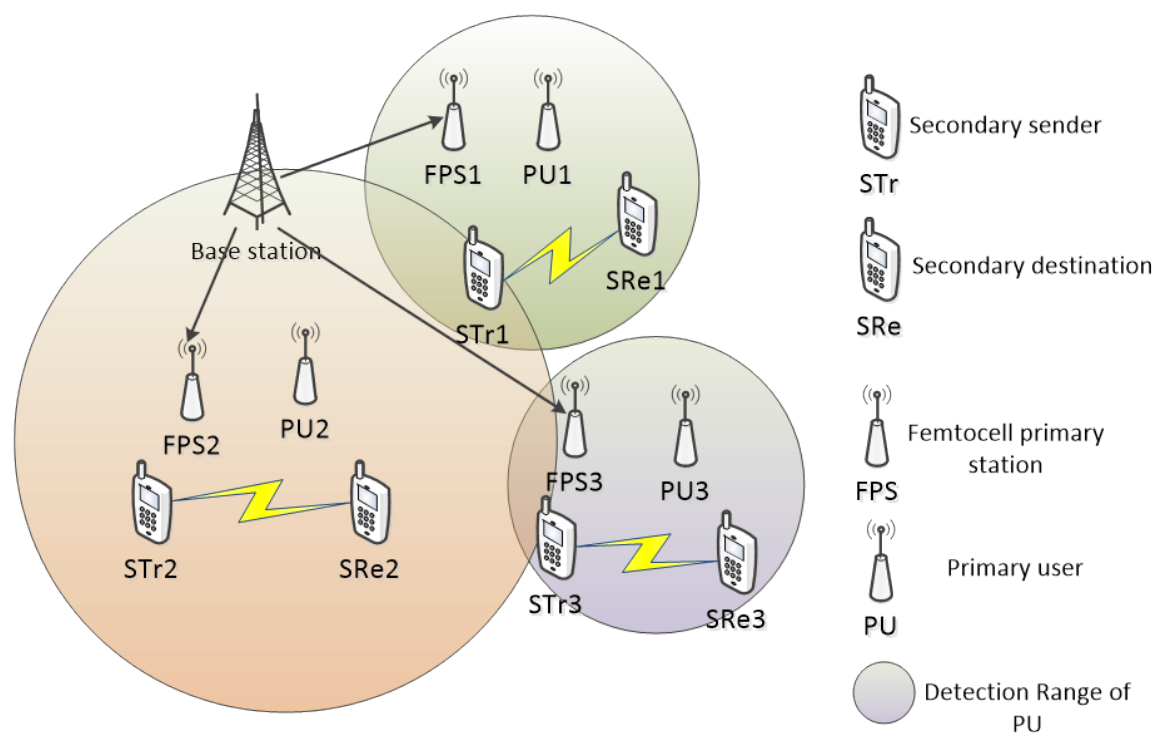

Fig. 1. System model

The cognitive heterogeneous networks which includes the primary base station (BS) and K primary femtocell is investigated, as depicted in Fig. 1. N secondary transmitter-receiver pairs (Tr-Re) sensing the status of the spectrum and access it, seperately. A licensed wideband OFDMA spectrum is considered to be divided into K non-overlapping narrowband channels that are allocated to $\mathrm{K}$ primary femtocell stations in the licensed network, $\boldsymbol{\kappa}_{j}$ denotes the set of subcarriers in the jth small cell which allocated to $\mathbf{P U}_{j}$ and one subcarrier can only be allocated to one PU, $\boldsymbol{\kappa}_{j} \cap \boldsymbol{\kappa}_{i}=\varnothing, \forall \boldsymbol{i} \neq \boldsymbol{j}$. Since PU may not occupy the licensed spectrum all the time, SU could access the spectrum according to the sensing results in the sensing- based spectrum sharing model. We assume that the channels are flat fading and the primary users' signals are complex-valued PSK signals. The noise is assumed to be independent and identically distributed circularly symmetric complex Gaussian (CSCG) with zero mean and $N_{0}$ variance.

In cognitive heterogeneous network, the primary user may only utilize a part of the licensed band rather than a whole network due to massive access. Thus, the small cell signal at PU always transmits with a weak power at around $10-15 \mathrm{~mW}$ and the transmission range of the PU signal is limited to only $150-200 \mathrm{~m}$. In this case, the detection range at SU is relatively small. To be more specific, if the location of SU is far from the PU in the jth small cell, the SU could not access the the jth channel. Only when the location of SU is within the detection range of $P U_{j}$, it can transmit data by accessing the jth subchannel and detect the status of $P U_{j}$. Therefore, each SU could access the corresponding set of the available channel according to the lotion of SU. For simplicity, this paper consider the case that only one secondary transmission pair and primary transmission pair exist in the jth small cell. Moreover, SU can access the ith subcarrier, where $\boldsymbol{g}_{i, j}^{p p}, \boldsymbol{g}_{i, j}^{s s}, \boldsymbol{g}_{i, j}^{s p}$ and $\boldsymbol{g}_{i, j}^{p s}$ denote the channel power gain of the primary link, the secondary link, the link between SU-Tx and PU-Rx and the link between PU-Tx and SU-Rx in the jth small cell. 
The energy detection scheme [19] is employed to perform simultaneous spectrum sensing for determining the status of each channel. The frame structure in this system consists of sensing slot $\tau$ and data transmission slot $T-\tau$, as shown in Fig. 2.

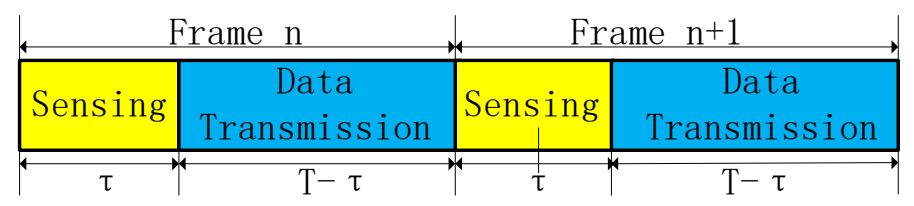

Fig. 2. Frame structure of the cognitive heterogeneous network

The detection of the channel status is a foremost task, which the frequency band is idle $\left(H_{0, j}\right)$ or active $\left(H_{1, j}\right)$. The probability of detection and false alarm for the jth channel is given by:

$$
\begin{aligned}
p_{d, j}\left(\tau, \varepsilon_{j}\right)= & Q\left(\left(\frac{\varepsilon_{j}}{N_{0}}-\gamma_{j}-1\right) \sqrt{\frac{\tau f_{s}}{2 \gamma_{j}+1}}\right), \\
p_{f a, j}\left(\tau, \varepsilon_{j}\right)= & Q\left(\left(\frac{\varepsilon_{j}}{N_{0}}-1\right) \sqrt{\tau f_{s}}\right) \\
& =Q\left(\sqrt{2 \gamma_{j}+1} Q^{-1}\left(p_{d, j}\right)+\sqrt{\tau f_{s}} \gamma_{j}\right) .
\end{aligned}
$$

Where $\varepsilon_{\mathrm{j}}$ is the decision threshold on the jth channel, $\gamma_{\mathrm{j}}$ represents the signal-to -noise ratio (SNR) of the primary user received at the secondary user on the jth channel, and $f_{\mathrm{s}}$ is the sampling frequency. The target detection probability is assumed to be $p_{d, j}\left(\tau, \varepsilon_{j}\right)=\bar{P}_{d, j}$.

\section{Optimal EE under the sensing-based rate loss constraint}

The sensing-based rate loss constraint is proposed in this section. The resource allocation optimal problems of energy efficiency are formulated with the sensing-based rate loss constraint and the average transmission power constraint for the sensing-based spectrum sharing model.

Table 1. Four possible scenarios for the SBSS model

\begin{tabular}{|c|c|c|c|c|}
\hline $\begin{array}{c}\text { PU's actual } \\
\text { status }\end{array}$ & $\begin{array}{c}\text { Decision } \\
\text { result }\end{array}$ & Related probability & Power & Rate \\
\hline Idle & $H_{0, j}$ & $P\left(H_{0, j}\right)\left(1-p_{f a, j}\left(\tau, \varepsilon_{j}\right)\right)$ & $P_{i, j}^{(s, 0)}$ & $r_{00, j}$ \\
\hline Idle & $H_{1, j}$ & $P\left(H_{0, j}\right) p_{f a, j}\left(\tau, \varepsilon_{j}\right)$ & $P_{i, j}^{(s, 1)}$ & $r_{01, j}$ \\
\hline Active & $H_{0, j}$ & $P\left(H_{1, j}\right)\left(1-p_{d, j}\left(\tau, \varepsilon_{j}\right)\right)$ & $P_{i, j}^{(s, 0)}$ & $r_{10, j}$ \\
\hline Active & $H_{1, j}$ & $P\left(H_{1, j}\right) p_{d, j}\left(\tau, \varepsilon_{j}\right)$ & $P_{i, j}^{(s, 1)}$ & $r_{11, j}$ \\
\hline
\end{tabular}

In order to achieve the higher spectrum efficiency, SU adapts its transmission power according to the detection results in the cognitive heterogeneous network. If the jth 
frequency band is detected to be idle $\left(H_{0, j}\right)$, SU transmits with high power $\left(P_{i, j}^{(s, 0)}\right)$, whereas if it is detected to be active $\left(H_{1, j}\right)$, SU transmits with low power $\left(P_{i, j}^{(s, 1)}\right)$. However, due to the limitation of the spectrum sensing techniques, the spectrum sensing is not a perfect function. Thus, according to the real state of PU and the sensing results, there are four possible scenarios for this model, as listed in the Table 1.

The four scenarios for the transmission state of SU in the abovementioned table are analyzed as follows:

If the spectrum is idle and PU is detected to be inactive, then SU will transmit with high power $P_{s, j}^{(0)}$. The probability of this scenario is $\alpha_{o, j}=P\left(H_{0, j}\right)\left(1-p_{f a, j}\left(\tau, \varepsilon_{j}\right)\right)$, where $P\left(H_{0, j}\right)$ denotes the probability that the jth spectrum band is idle. The instantaneous transmission capacity is:

$$
r_{00, j}=\sum_{i \in K_{j}} \log _{2}\left(1+\frac{g_{s s, i} P_{i, j}^{(s, 0)}}{N_{0}}\right),
$$

If the spectrum is idle but PU is detected to be active, then SU will transmit with low power $P_{s, j}^{(1)}$. The probability of this scenario is $\alpha_{1, j}=P\left(H_{0, j}\right) p_{f a, j}\left(\tau, \varepsilon_{j}\right)$, and the instantaneous transmission capacity is :

$$
r_{01, j}=\sum_{i \in \mathrm{K}_{j}} \log _{2}\left(1+\frac{g_{s s, i} P_{i, j}^{(s, 1)}}{N_{0}}\right)
$$

If the spectrum is busy and PU is detected to be inactive, then SU will transmit with high power $P_{s, j}^{(0)}$. The probability of this scenario is $\beta_{0, j}=P\left(H_{1, j}\right)\left(1-p_{d, j}\left(\tau, \varepsilon_{j}\right)\right)$ where $P\left(H_{1, j}\right)$ denotes the probability that the jth spectrum band is active. The instantaneous transmission capacity is:

$$
r_{10, j}=\sum_{i \in \mathrm{K}_{j}} \log _{2}\left(1+\frac{g_{s s, i} i_{i, j}^{(s, 0)}}{g_{p s, i} P_{p, i}+N_{0}}\right),
$$

If the spectrum is busy and PU is detected to be active, then SU will transmit with low power $P_{s, j}^{(1)}$. The probability of this scenario is $\beta_{1, j}=P\left(H_{1, j}\right) p_{d, j}\left(\tau, \varepsilon_{j}\right)$. The instantaneous transmission capacity is:

$$
r_{11, j}=\sum_{i \in \mathrm{K}_{j}} \log _{2}\left(1+\frac{g_{s s, i} P_{i, j}^{(s, 1)}}{g_{p s, i} P_{p, i}+N_{0}}\right) .
$$

To effectively protect the QoS of PU, the sensing-based rate loss constraint based on the detection results in the SBSS model is proposed. The rate loss of PU caused by SU's transmission occurred in nothing but the data transmission slot, so we only focus on the data transmission slot in the following studies.

When the SU could not access the licensed band, the transmission rate of $\mathrm{PU}_{\mathrm{j}}$ in the data transmission slot $T-\tau$ on the jth channel is given by

$$
R_{j}^{p}=\frac{T-\tau}{T} \sum_{i \in K_{j}} P\left(H_{1, j}\right) \log _{2}\left(1+\frac{g_{p p, i} P_{p, i}}{N_{0}}\right), \forall j \in\{1,2, \ldots, M\} .
$$

Where $P_{p, i}$ represents the transmission power of the jth PU.

When the SU access the licensed band, the PU will suffer from the potential interference. The interference to PU may occur in two cases: 1) missed detection, when the PU is falsely 
detected to be idle; 2) correct detection, when the PU is correctly detected to be active. As a result, the average rate of $\mathrm{PU}_{\mathrm{j}}$ can be formulated as:

$$
R_{j}^{s}=\frac{T-\tau}{T} \sum_{i \in K_{j}} \beta_{0, j} \log _{2}\left(1+\frac{g_{p p, i} P_{p, i}}{N_{0}+g_{s p, i} P_{i, j}^{(s, 0)}}\right)+\beta_{1, j} \log _{2}\left(1+\frac{g_{p p, i} P_{p, i}}{N_{0}+g_{s p, i} P_{i, j}^{(s, 1)}}\right), \forall j \in\{1,2, \ldots, M\} .
$$

Then the average rate loss of $\mathrm{PU}_{\mathrm{j}}$ can be expressed $R_{j}^{p}-R_{j}^{s}$, and the sensing-based rate loss constraint under the imperfect spectrum sensing for SBSS model in cognitive heterogeneous network can be defined as

$$
R_{j}^{p}-R_{j}^{s} \leq \Delta R_{j}, \forall j \in\{1,2, \ldots, \mathrm{M}\},
$$

Where $\Delta R_{j}$ represents the maximum rate loss that PU can tolerate.

Let $R_{j}=R_{j}^{p}-\Delta R_{j}, \forall j \in\{1,2, \ldots, \mathrm{M}\}$, the sensing-based rate loss constraint can be rewritten as

$$
R_{j}^{s} \geq R_{j}, \forall j \in\{1,2, \ldots, \mathrm{M}\} .
$$

Furthermore, the average transmission power constraint should be taken into account to keep long-term transmission. The average transmit power constraint can be given by:

$$
\frac{T-\tau}{T} \sum_{j=1}^{M} \sum_{i \in K_{j}}\left(\alpha_{o, j}+\beta_{0, j}\right) P_{i, j}^{(s, 0)}+\left(\alpha_{1, j}+\beta_{1, j}\right) P_{i, j}^{(s, 1)} \leq P_{a v},
$$

Where $P_{a v}$ represents the maximum average transmission power of SU.

The average rate of the jth SU for the SBSS model can be expressed as:

$$
C_{j}\left(\tau, P_{s}^{(0)}, P_{s}^{(1)}\right)=\frac{T-\tau}{T}\left(\alpha_{o, j} r_{00, j}+\alpha_{1, j} r_{01, j}+\beta_{0, j} r_{10, j}+\beta_{1, j} r_{11, j}\right) .
$$

In the sensing slot, the energy consumption for SU transmission is:

$$
E_{s}(\tau)=\tau P_{s}^{(s)},
$$

Where $P_{s}^{(s)}$ denotes the power consumption of sensing.

In the transmission slot, the energy consumption for SU transmission is:

$$
E_{S}\left(\tau, P_{s}^{(0)}, P_{s}^{(1)}\right)=(T-\tau) \sum_{j=1}^{M} \sum_{i \in K_{j}}\left(\alpha_{o, j}+\beta_{0, j}\right) P_{i, j}^{(s, 0)}+\left(\alpha_{1, j}+\beta_{1, j}\right) P_{i, j}^{(s, 1)},
$$

Then the energy efficiency for the SBSS model in cognitive heterogeneous network with "bite per joule" is:

$$
U_{E E}\left(\tau, P_{s}^{(0)}, P_{s}^{(1)}\right)=\frac{\sum_{j=1}^{M} C_{j}\left(\tau, P_{s}^{(0)}, P_{s}^{(1)}\right)}{E_{S}\left(\tau, P_{s}^{(0)}, P_{s}^{(1)}\right)+E_{S}(\tau)+E_{C}},
$$

Where $E_{C}$ is the circuit power consumption derived from signal processing, battery backup, and others.

Thus, the energy efficiency resource allocation with imperfect sensing results under the sensing based rate loss constraint and transmission power constraint in cognitive heterogeneous networks can be formulated as follows:

$$
\max _{\left\{\tau, P_{s}^{(0)}, P_{s}^{(1)}\right\}} U_{E E}\left(\tau, P_{s}^{(0)}, P_{s}^{(1)}\right) \text {, }
$$

Subject to (10), (11), $P_{i, j}^{(s, 0)} \geq 0, P_{i, j}^{(s, 1)} \geq 0,0 \leq \tau \leq T, j=1, \ldots, M$. 
Due to the nonconvex problem of the objective function, we could not employ the convex optimization algorithm to obtain the optimal sensing time directly. However, considering the sensing time lies within the interval $(0 \square \mathrm{T} \square$, the optimal sensing time can be acquired by one-dimensional exhaustive search as follows:

$$
\tau^{*}=\arg \max U_{E E}\left(\tau, P_{s}^{(0)}, P_{s}^{(1)}\right) .
$$

Lemma 1. Function $U_{E E}\left(\tau^{*}, P_{s}^{(0)}, P_{s}^{(1)}\right)$ is strictly quasi-concave in $P_{i, j}^{(s, 0)}, P_{i, j}^{(s, 1)}$, respectively.

\section{Proof: See Appendix A.}

Accordingly, the strictly quasi-concave function $U_{E E}\left(\tau^{*}, P_{s}^{(0)}, P_{s}^{(1)}\right)$ has a unique globally optimal transmission power.

The optimal energy efficiency problem can be associated with the following function problem $\mathrm{F}(q)$ for the optimal sensing time $\tau^{*}$ :

$$
\max _{P_{P^{(s, j)}}^{(,)}, P_{P_{j}, j}^{(,)}} \sum_{j=1}^{M} C_{j}\left(\tau^{*}, P_{s}^{(0)}, P_{s}^{(1)}\right)-q\left(E_{S}\left(\tau^{*}, P_{s}^{(0)}, P_{s}^{(1)}\right)+E_{S}\left(\tau^{*}\right)+E_{C}\right),
$$

where $q \in R$ is a parameter.

Theorem 1. $\left(P_{i, j}^{(s, 0), *}, P_{i, j}^{(s, 1), *}\right)$ is the optimal solution of (16) associated with the maximum value $q^{*}$ if and only if $\mathrm{F}\left(q^{*}\right)=\mathrm{F}\left(q^{*}, P_{i, j}^{(s, 0), *}, P_{i, j}^{(s, 1), *}\right)=0$.

\section{Proof: See Appendix B.}

The equivalent objective function is convex with respect to the transmit powers $P_{i, j}^{(s, 0)}$ and $P_{i, j}^{(s, 1)}$. However, equation (11) makes it a non-convex optimization problem, so the optimal problem cannot be solved by considering the Lagrange dual problem. To solve the problem, the lemma is given as follows.

Lemma 2. The equivalent objective function optimization problem satisfies the Time-Sharing Property when subcarriers $\kappa_{j}$ goes to infinity.

\section{Proof: See Appendix C.}

The time sharing property implies that the duality gap for the equivalent optimization problem is negligible with realistic number of subcarriers and it can be solved by the Lagrange dual method.

The Lagrangian function of $\mathrm{F}(q)$ with respect to $\left(P_{i, j}^{(s, 0)}, P_{i, j}^{(s, 1)}\right)$ for a fixed sensing time is derived as:

$$
\begin{aligned}
& L\left(P_{i, j}^{(s, 0)}, P_{i, j}^{(s, 1)}, \lambda, \mu\right)=\frac{T-\hat{\tau}}{T} \sum_{j=1}^{M}\left(\alpha_{o, j} r_{00, j}+\alpha_{1, j} r_{01, j}+\beta_{0, j} r_{10, j}+\beta_{1, j} r_{11, j}\right)-q\left(E_{S}\left(\hat{\tau}, P_{s}^{(0)}, P_{s}^{(1)}\right)+E_{S}(\hat{\tau})+E_{C}\right) \\
& -\lambda\left(\frac{T-\hat{\tau}}{T} \sum_{j=1}^{M} \sum_{i \in K_{j}}\left(\alpha_{o, j}+\beta_{0, j}\right) P_{i, j}^{(s, 0)}+\left(\alpha_{1, j}+\beta_{1, j}\right) P_{i, j}^{(s, 1)}-P_{a v}\right)+\sum_{j=1}^{M} \mu_{j}\left(R_{j}^{s}-R_{j}\right),
\end{aligned}
$$

Where $\lambda$ and $\mu_{j}$ are the lagrangian multipliers. The dual objective function can be expressed as:

Where

$$
\begin{aligned}
& g(\lambda, \boldsymbol{\mu})=\max _{P_{l, j}^{(s, j)}, P_{i, j}^{(s, 1)}} L\left(P_{i, j}^{(s, 0)}, P_{i, j}^{(s, 1)}, \lambda, \mu\right) \\
& =\max _{P_{l, j}^{(s, 0)}, P_{l, j}^{(s)}} \tilde{g}(\lambda, \boldsymbol{\mu})-q\left(E_{S}(\hat{\tau})+E_{C}\right)+\lambda P_{a v}-\sum_{j=1}^{M} \mu_{j} R_{j},
\end{aligned}
$$




$$
\begin{aligned}
& \tilde{g}(\lambda, \boldsymbol{\mu})=\frac{T-\hat{\tau}}{T} \sum_{j=1}^{M}\left(\alpha_{o, j} r_{00, j}+\alpha_{1, j} r_{01, j}+\beta_{0, j} r_{10, j}+\beta_{1, j} r_{11, j}\right)-q E_{S}\left(\hat{\tau}, P_{s}^{(0)}, P_{s}^{(1)}\right) \\
& -\lambda\left(\frac{T-\hat{\tau}}{T} \sum_{j=1}^{M} \sum_{i \in K_{j}}\left(\alpha_{o, j}+\beta_{0, j}\right) P_{i, j}^{(s, 0)}+\left(\alpha_{1, j}+\beta_{1, j}\right) P_{i, j}^{(s, 1)}\right) \\
& +\frac{T-\tau}{T} \sum_{j=1}^{M} \mu_{j} \sum_{i \in K_{j}} \beta_{0, j} \log _{2}\left(1+\frac{g_{p p, i} P_{p, i}}{N_{0}+g_{s p, i} P_{i, j}^{(s, 0)}}\right)+\beta_{1, j} \log _{2}\left(1+\frac{g_{p p, i} P_{p, i}}{N_{0}+g_{s p, i} P_{i, j}^{(s, 1)}}\right) .
\end{aligned}
$$

For a fixed sensing time, the equalization optimization problem respecting to the transmission power $P_{i, j}^{(s, 0)}$ and $P_{i, j}^{(s, 1)}$ is convex, respectively. So we can solve the problem by using the dual decomposition method. In the following, it is observed that the joint optimization problem can be decomposed into two optimization subproblems, SP1 and SP2.

SP1:

$$
\max _{P_{i, j}^{(s, 0)} \geq 0} \frac{T-\hat{\tau}}{T} \sum_{j=1}^{M}\left(\alpha_{o, j} r_{00, j}+\beta_{0, j} r_{10, j}\right)-\left(q+\frac{\lambda}{T}\right)(T-\hat{\tau}) \sum_{j=1}^{M} \sum_{i \in K_{j}}\left(\alpha_{o, j}+\beta_{0, j}\right) P_{i, j}^{(s, 0)}
$$

$$
\begin{aligned}
& +\frac{T-\hat{\tau}}{T} \sum_{j=1}^{M} \mu_{j} \sum_{i \in \mathrm{K}_{j}} \beta_{0, j} \log _{2}\left(1+\frac{g_{p p, i} P_{p, i}}{N_{0}+g_{s p, i} P_{i, j}^{(s, 0)}}\right), \\
& \max _{P_{i, j}^{(s)} \geq 0} \frac{T-\hat{\tau}}{T} \sum_{j=1}^{M}\left(\alpha_{1, j} r_{01, j}+\beta_{1, j} r_{11, j}\right)-\left(q+\frac{\lambda}{T}\right)(T-\hat{\tau}) \sum_{j=1}^{M} \sum_{i \in \mathrm{K}_{j}}\left(\alpha_{1, j}+\beta_{1, j}\right) P_{i, j}^{(s, 1)}
\end{aligned}
$$

SP2:

$$
+\frac{T-\hat{\tau}}{T} \sum_{j=1}^{M} \mu_{j} \sum_{i \in \mathrm{K}_{j}} \beta_{1, j} \log _{2}\left(\frac{g_{p p, i} P_{p, i}}{1+\frac{\left.N_{0}+g_{S p, i} P_{i, j}, 1\right)}{N}}\right) .
$$

By giving their Lagrangian functions and employing the Karush-Kuhn-Tucher (KKT) conditions, the optimal power allocation is obtained by the theorems as follows:

Theorem 2. The optimal transmission power under the sensing-based rate loss constraint equals to $\left\{P_{i, j}^{(s, 0)}, 0\right\}$, where $P_{i, j}^{(s, 0)}$ is the solution of

$$
\frac{\alpha_{o, j} g_{s s, i}}{1+g_{s s, i} P_{i, j}^{(s, 0)}}+\frac{\beta_{0, j} g_{s s, i}}{g_{p s, i} P_{p, i}+N_{0}+g_{s s, i} P_{i, j}^{(s, 0)}}=(q T+\lambda)\left(\alpha_{o, j}+\beta_{0, j}\right)+\frac{\mu_{j} \beta_{0, j} g_{p p, i} g_{s p, i} P_{p, i}}{\left(N_{0}+g_{p p, i} P_{p, i}+g_{s p, i} P_{i, j}^{(s, 0)}\right)\left(N_{0}+g_{s p, i} P_{i, j}^{(s, 0)}\right)} \text {. }
$$

Theorem 3. The optimal transmission power under the sensing-based rate loss constraint equals to $\left\{P_{i, j}^{(s, 1)}, 0\right\}$, where $P_{i, j}^{(s, 1)}$ is the solution of

$$
\frac{\alpha_{1, j} g_{s s, i}}{1+g_{s s, i} P_{i, j}^{(s, 1)}}+\frac{\beta_{1, j} g_{s s, i}}{g_{p s, i} P_{p, i}+N_{0}+g_{s s, i} P_{i, j}^{(s, 1)}}=(q T+\lambda)\left(\alpha_{1, j}+\beta_{1, j}\right)+\frac{\mu_{j} \beta_{1, j} g_{p p, i} g_{s p, i} P_{p, i}}{\left(N_{0}+g_{p p, i} P_{p, i}+g_{s p, i} P_{i, j}^{(s, 1)}\right)\left(N_{0}+g_{s p, i} P_{i, j}^{(s, 1)}\right)} .
$$

According to the Theorem 2 and 3 , the transmission power $\left(P_{i, j}^{(s, 0)}, P_{i, j}^{(s, 1)}\right)$ for fixed q, $\lambda$ and $\mu_{j}$ could not obtained directly. The bisection search method should be used to solve the problem. However, the bisection search range of the transmit power should be identified.

Proportion 1. the transmission power $P_{i, j}^{(s, 0)}$ for fixed q, $\lambda$ and $\mu_{j}$ exists in the range of $[0, \phi]$, where $F(\phi)=(q T+\lambda)\left(\alpha_{o, j}+\beta_{0, j}\right)$ only if the left hand side function and the right hand side function in the Theorem 2 satisfying $F(0) \geq H(0)$. 


\section{Proof: See Appendix D.}

The transmission power in the theorem $3 P_{i, j}^{(s, 1)}$ can also be obtained by using the same method. The energy efficiency optimal algorithm is given by the following subgradient algorithm.

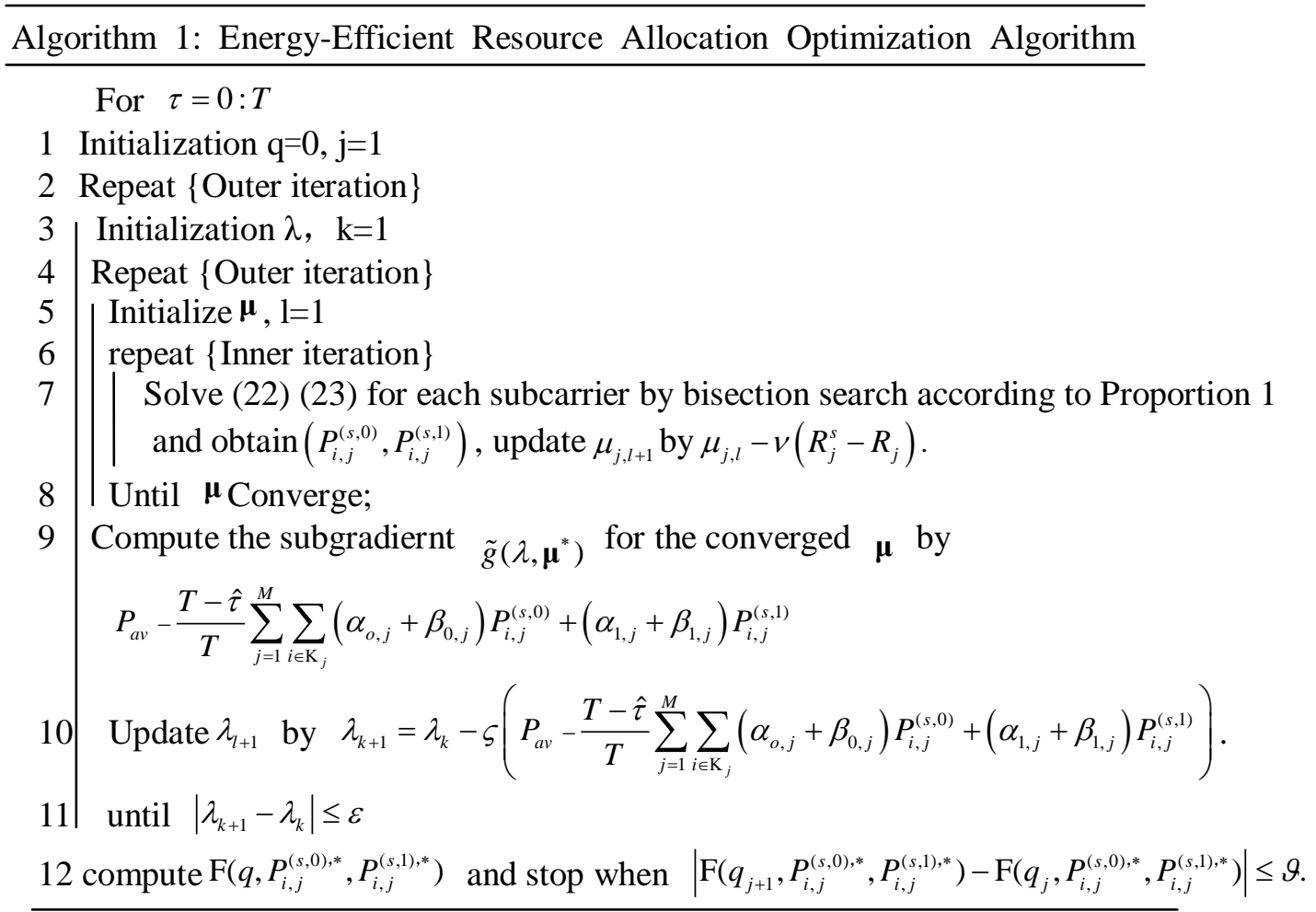

For obtaining the optimal transmission power $\left(P_{i, j}^{(s, 0)}, P_{i, j}^{(s, 1)}\right)$, we proposed an optimal solution in Theorem 2 and 3 which is solved by exhaustive search and hence has a very high complexity. Furthermore, the bisection search range of the transmit power is proved in Proposition 1 to reduce the complexity. According to the abovementioned algorithm 1, the transmission power $P_{i, j}^{(s, 0)}$ and $P_{i, j}^{(s, 1)}$ in Theorem 2 and 3 should be obtained by using bisection method with the complexity $o\left(2 \log _{2}(\delta)\right)$, where $\delta$ is the required accuracy. The updates of lagrange multipliers $\lambda$ and $\mu$ in each iteration of the algorithm 1 for each secondary transmission pair on each subcarrier need $o\left(2 K M \log _{2}(\delta)\right)$. As a result, the complexity of the proposed energy efficiency optimal algorithm is $o\left(2 T_{\mathrm{m}} K^{2} M^{2} \log _{2}(\delta)\right)$, where $T_{\mathrm{m}}$ is the maximum iteration times and a polynomial function of $K^{2} M^{2}$. Additionally, $T_{\mathrm{m}}$ can be very small when the initial values of lagrange multipliers $\lambda$ and $\boldsymbol{\mu}$ are well chosen.

\section{Optimal EE under the conventional interference power constraint}

In this section, we use the conventional interference power constraint to protect PU in the same system model. However, due to the limitation of spectrum sensing techniques, it is not 
evitable that there would be a case of missed detection. Then the conventional interference power constraint under imperfect sensing results can be written as

$$
\frac{T-\tau}{T} \sum_{j=1}^{M} \sum_{i \in \mathrm{K}_{j}} \beta_{0, j} g_{s p, i} P_{i, j}^{(s, 0)}+\beta_{1, j} g_{s p, i} P_{i, j}^{(s, 1)} \leq \Gamma, j=1, \ldots, M,
$$

Where $\Gamma$ is the maximum interference power that the PU could tolerate.

To keep the long-term power budget of SU, the transmit power constraint is also used. Then, it is given by (11) in section III.

Thus, the maximum energy efficiency for the SBSS model under the conventional interference constraint can be formulated as:

$$
U_{E E}\left(\tau, P_{s}^{(0)}, P_{s}^{(1)}\right)=\frac{\sum_{j=1}^{M} C_{j}\left(\tau, P_{s}^{(0)}, P_{s}^{(1)}\right)}{E_{S}\left(\tau, P_{s}^{(0)}, P_{s}^{(1)}\right)+E_{S}(\tau)+E_{C}},
$$

Subject to (11), (23), $P_{i, j}^{(s, 0)} \geq 0, P_{i, j}^{(s, 1)} \geq 0, \quad 0 \leq \tau \leq T, j=1, \ldots, M$.

The optimal sensing time also could not be obtained directly by the convex optimization algorithm. So the one-dimensional exhaustive search method could be employed to solve the sensing time optimization problem. Furthermore, we focus on finding the optimal power allocation $\left(P_{i, j}^{(s, 0) *}, P_{i, j}^{(s, 1) *}\right)$ that maximizes the energy efficiency under the conventional interference constraint.

However, The energy efficiency optimal problem can also be transformed into a equivalent function problem $\mathrm{F}(q)$ for the fixed sensing time $\tau$. Then, by giving the Lagrangian $L\left(P_{s}^{(0)}, P_{s}^{(1)}, \lambda, \mu\right)$ and employing the KKT condition the optimal transmission power under the conventional interference constraint could be expressed in the two following theorems.

Theorem 4. The optimal transmission power when the spectrum is detected to be idle can be obtained as:

$$
P_{i, j}^{(0)}=\left[\frac{B_{i, j}^{(0)}+\sqrt{\Delta_{i, j}^{(0)}}}{2 A_{i, j}^{(0)}}\right]^{+},
$$

where $[\mathrm{x}]^{+}$denotes $\max (\mathrm{x}, 0)$,

$$
\begin{aligned}
& A_{i, j}^{(0)}=\left[(q T+\lambda)\left(\alpha_{0, j}+\beta_{0, j}\right)+\mu_{j} \beta_{0, j} g_{s p p i}\right] g_{s s, i}^{2}, \\
& B_{i, j}^{(0)}=\left(\alpha_{0, j}+\beta_{0, j}\right) g_{s s, i}^{2}-\left[(q T+\lambda)\left(\alpha_{0, j}+\beta_{0, j}\right)+\mu_{j} \beta_{0, j} g_{s p, i}\right]\left(g_{p s, i} P_{p, i}+2 N_{0}\right) g_{s s, i}, \\
& \Delta_{i, j}^{(0)}=\left(B_{i, j}^{(0)}\right)^{2}-4 A_{i, j}^{(0)} C_{i, j}^{(0)}, \\
& C_{i, j}^{(0)}=\left[(q T+\lambda)\left(\alpha_{0, j}+\beta_{0, j}\right)+\mu_{j} \beta_{0, j} g_{s p, i}\right] N_{0}\left(g_{p s, i} P_{p, i}+N_{0}\right)-\left(\alpha_{0, j} g_{s s, i}\left(g_{p s, i} P_{p, i}+N_{0}\right)+\beta_{0, j} g_{s s, i} N_{0}\right) .
\end{aligned}
$$

Theorem 5. The optimal transmission power when the spectrum is detected to be active can be obtained as:

$$
P_{i, j}^{(1)}=\left[\frac{B_{i, j}^{(1)}+\sqrt{\Delta_{i, j}^{(1)}}}{2 A_{i, j}^{(1)}}\right]^{+} .
$$

where 
$A_{i, j}^{(1)}=\left[(q T+\lambda)\left(\alpha_{1, j}+\beta_{1, j}\right)+\mu_{j} \beta_{1, j} g_{s p, i}\right] g_{s s, i}^{2}$,

$B_{i, j}^{(1)}=\left(\alpha_{1, j}+\beta_{1, j}\right) g_{s s, i}^{2}-\left[(q T+\lambda)\left(\alpha_{1, j}+\beta_{1, j}\right)+\mu_{j} \beta_{1, j} g_{s p, i}\right]\left(g_{p s, i} P_{p, i}+2 N_{0}\right) g_{s s, i}$,

$\Delta_{i, j}^{(1)}=\left(B_{i, j}^{(1)}\right)^{2}-4 A_{i, j}^{(1)} C_{i, j}^{(1)}$,

$C_{i, j}^{(1)}=\left[(q T+\lambda)\left(\alpha_{1, j}+\beta_{1, j}\right)+\mu_{j} \beta_{1, j} g_{s p, i}\right] N_{0}\left(g_{p s, i} P_{p, i}+N_{0}\right)-\left(\alpha_{1, j} g_{s s, i}\left(g_{p s, i} P_{p, i}+N_{0}\right)+\beta_{1, j} g_{s s, i} N_{0}\right)$.

According to Theorem 1 , the optimal transmission power $\left(P_{i, j}^{(s, 0) *}, P_{i, j}^{(s, 1) *}\right)$ under the conventional interference constraint is obtained by finding the root for the equivalent function, $\mathrm{F}\left(q^{*}, P_{k, i}^{(0), *}, P_{k, i}^{(1) * *}\right)=0$. For fixed $\lambda$ and $\mu_{j}$, the resource allocation optimal strategy for the maximum energy efficiency is obtained.

\section{Relationship between the two constraints with imperfect sensing}

The sensing-based rate loss constraint for cognitive heterogeneous networks is derived. However, the relationship between the proposed protection criterion and the conventional interference constraint with imperfect spectrum sensing results is necessary to be investigated. In this section, the interference power constraint is proved that could serve as an upper bound of the maximum rate loss that PU could tolerate in SBSS model.

The per subcarrier based interference power constraint at the jth PU is written as:

$$
\frac{T-\tau}{T}\left(g_{s p, i} \beta_{0, j} P_{i, j}^{(s, 0)}+g_{s p, i} \beta_{1, j} P_{i, j}^{(s, 1)}\right) \leq \Gamma_{i, j}, i \in \mathrm{K}_{j}, j \in\{1, \ldots, N\} .
$$

Where $\Gamma_{i, j}$ denotes the maximum interference power that $P U_{\mathrm{j}}$ in ith subcarrier can tolerate. Theorem 6. The maximum rate loss of $P U_{j}$ based imperfect spectrum sensing results is upper-bounded by $\frac{K_{j}}{N_{0} \ln 2} \Gamma_{i, j}$, if the interference threshold of the ith subcarrier $\Gamma_{i, j}$ exists at the jth PU as (28).

Proof: See Appendix E.

Therefore, the sensing based rate loss constraint

$$
R_{j}^{p}-R_{j}^{s} \leq \frac{\mathrm{K}_{j}}{N_{0} \ln 2} \Gamma_{i, j} .
$$

It is seen from the above proof, the maximum rate loss of PU is upper-bounded by $\frac{K_{j}}{N_{0} \ln 2} \Gamma_{i, j}$. If the upper bound meets $\frac{\mathrm{K}_{j}}{N_{0} \ln 2} \Gamma_{i, j} \leq \Delta R_{j}$, then the sensing based rate loss constraint is clearly satisfied. Therefore, if we choose the interference threshold $\Gamma_{i, j}=N_{0} \ln 2 \frac{\Delta R_{j}}{\mathrm{~K}_{j}}$, the rate loss at the jth PU would be less than $\Delta R_{j}$.

\section{Simulation results}

The optimal system performance of SBSS cognitive heterogeneous network under the two above-mentioned constraint schemes is numerically evaluated in this section, respectively. The channel power gains are assumed to be exponentially distributed random variables with unit mean. The noise variance is set to 1 . The frame duration is chosen to be $T=100$ $\mathrm{ms}$, and the transmit power of $\mathrm{PU}$ is assumed to be $10 \mathrm{~dB}$. The number of the subcarriers 
is 128 . The bandwidth of each subcarrier is $10 \mathrm{KHz}$. The circuit power and the sensing power are set to be $0.4 \mathrm{~W}$ and $0.2 \mathrm{~W}$, respectively.

Fig. 3 presents energy efficiency versus the sensing time of SU for several values of the transmission power constraint $P_{a v}$ under the two above-mentioned constraint schemes, the proposed protection criterion and the conventional interference constraint. The rate loss threshold that PU can tolerate is set to be $20 \%$ and the probability that the jth channel is idle is $P\left(H_{0, j}\right)=0.6$. For the conventional power constraint based on the per subcarrier case, the interference thresholds are chosen as $\Gamma_{i, j}=N_{0} \ln 2 \Delta R_{j} / \mathrm{K}_{j}$ which guarantees the fairness that the upper bounds of PU's rate loss are the same as the proposed protection constraint case. It is observed that the energy efficiency is convex with respect to the sensing time and the optimal sensing time for all four sets of parameters is almost around $7 \mathrm{~ms}$ under the sensing based rate loss constraint. The energy efficiency also increases with $P_{a v}$ under interference power constraint, but the optimal sensing time for the four sets of parameters is almost around 10 ms. As seen from Fig. 4, the energy efficiency under the two constraint schemes increases with $P_{a v}$ and the difference of the energy efficiency is obvious, when $P_{a v}$ is large. This is because that the sensing-based rate loss constraint gradually becomes the dominant constraint with the increase of $P_{a v}$. The optimal sensing time under the sensing-based rate loss constraint is slightly smaller than that under the interference power constraint and slightly increases with $P_{a v}$ increasing. Therefore, the sensing-based rate loss constraint is more suitable for the SBSS model than the interference power constraint.

In Fig. 4, the EE versus the rate loss of PU is presented for various values of the number of SU under different sensing scenarios. The sensing scenarios include two cases: (1) perfect sensing when the SBS correctly senses the status of PU, $P_{d}=1, P_{f}=0$; (2) imperfect sensing due to the limitation of sensing technology results in missed detection and false alarm, $P_{d}<1$. The maximum average transmit power $P_{a v}$ is set to be 5 $\mathrm{dB}$, and the probability of false alarm $P_{f}$ is 0.001 . The sensing time is assumed to be the optimal sensing time, which is $7 \mathrm{~ms}$. Owing to the reality of false alarm and misdetection, EE in perfect sensing scenario is always higher than that in imperfect sensing scenario. Fig. 4 shows that EE increases with the number of SU increasing. EE increases slowly when the rate loss constraint threshold becomes higher in value. This condition is reasonable because the interference power constraint is not the main limit for the maximum EE when the interference power constraint threshold is a higher power.

In Fig. 5, the EE versus the interference power constraint threshold $\Gamma$ is presented for various values of the probability $\mathrm{P}\left(\mathrm{H}_{0}\right)$ under the two constraint schemes, the proposed protection criterion and the conventional interference constraint. The sensing time is set to be $7 \mathrm{~ms}$ and the probability of false alarm $P_{f}$ is assumed as 0.001 . The maximum average transmission power threshold is assumed as $\mathrm{P}_{a v}=5 d B$. For fair comparison, the interference thresholds are chosen as $\Delta R_{j}=\Gamma_{i, j} \mathrm{~K}_{j} / N_{0} \ln 2$. EE under the two conditions increases with the interference constraint respectively. However, EE does not increase when the interference power constraint threshold $\Gamma$ becomes larger since the interference power constraint would not be the dominating constraint for the EE when it becomes larger. But EE increases with the interference power constraint threshold $\Gamma$ becoming larger. For the same probability $\mathrm{P}\left(\mathrm{H}_{0}\right)$, the EE under the sensing-based rate loss constraint is always higher than that under the interference power constraint. This finding is attributed to the facts that SU under the sensing-based rate loss constraint could adjust transmission power according to the transmission rate of PU. However, when the 
probability $\mathrm{P}\left(\mathrm{H}_{0}\right)$ is 0.9 , the EE under the sensing-based rate loss constraint is slightly larger than that under the conventional interference power constraint. This finding is attributed to the facts that when the probability $\mathrm{P}\left(\mathrm{H}_{0}\right)$ is higher, the sensing-based rate loss constraint is almost same as the conventional interference power constraint. However, the proposed rate loss constraint is more effective compared with the conventional constraint.

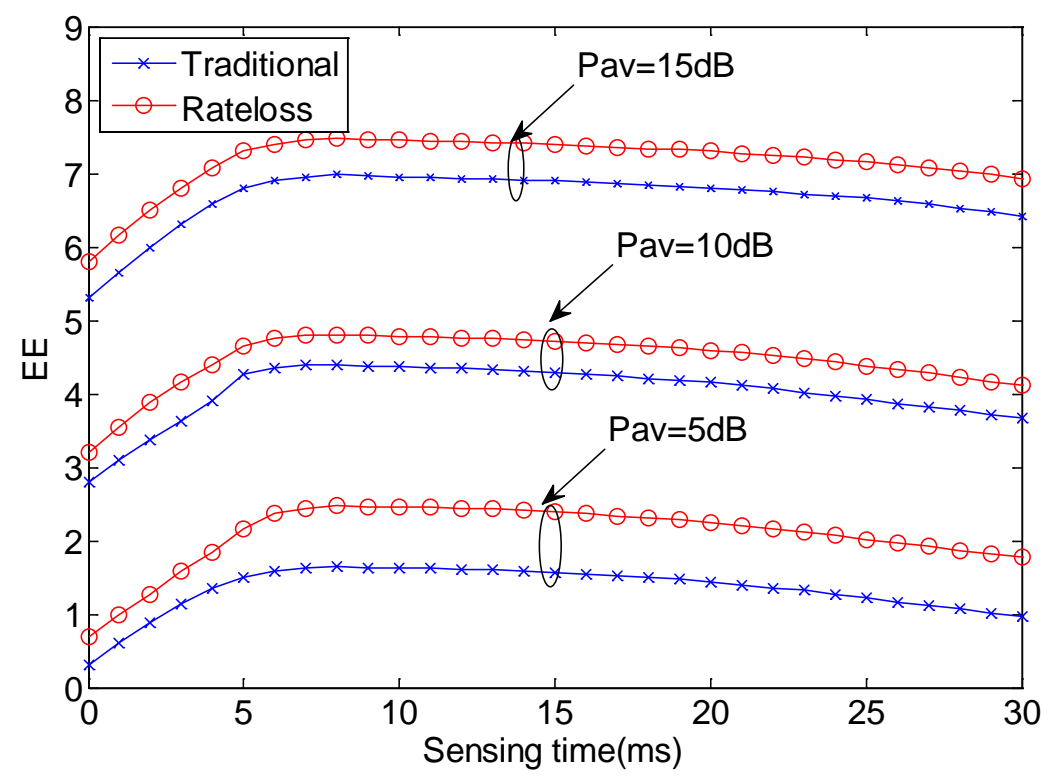

Fig. 3. The EE versus the sensing time under different interference constraints

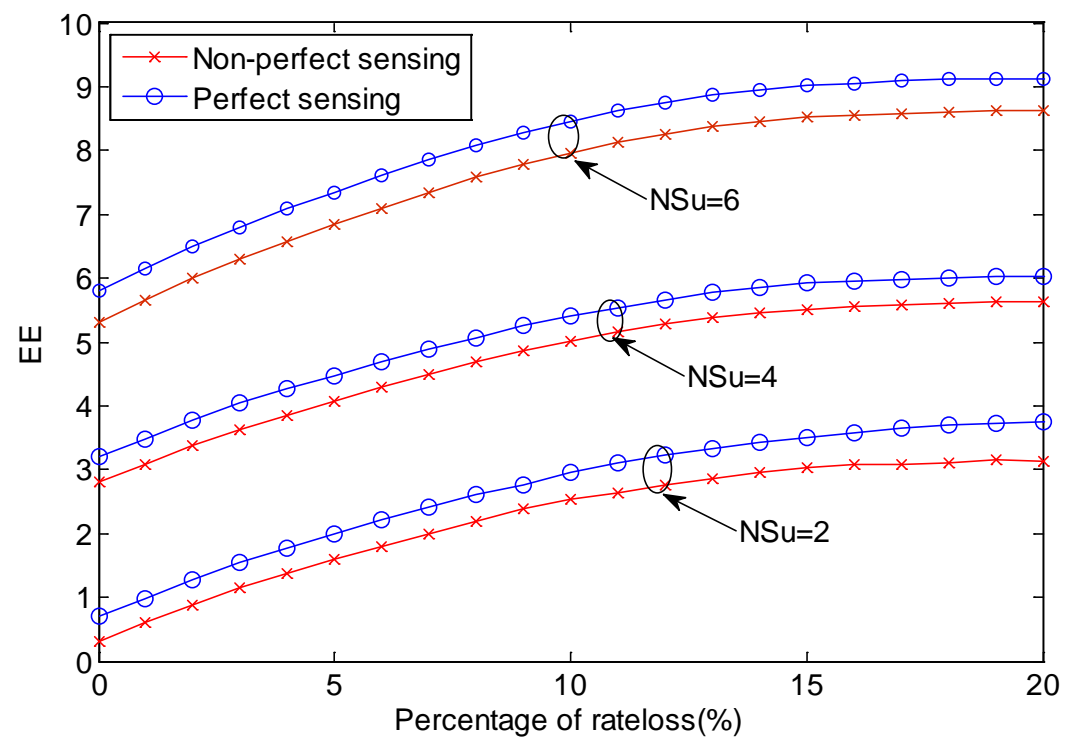

Fig. 4. The EE versus rate loss under different sensing scenarios 


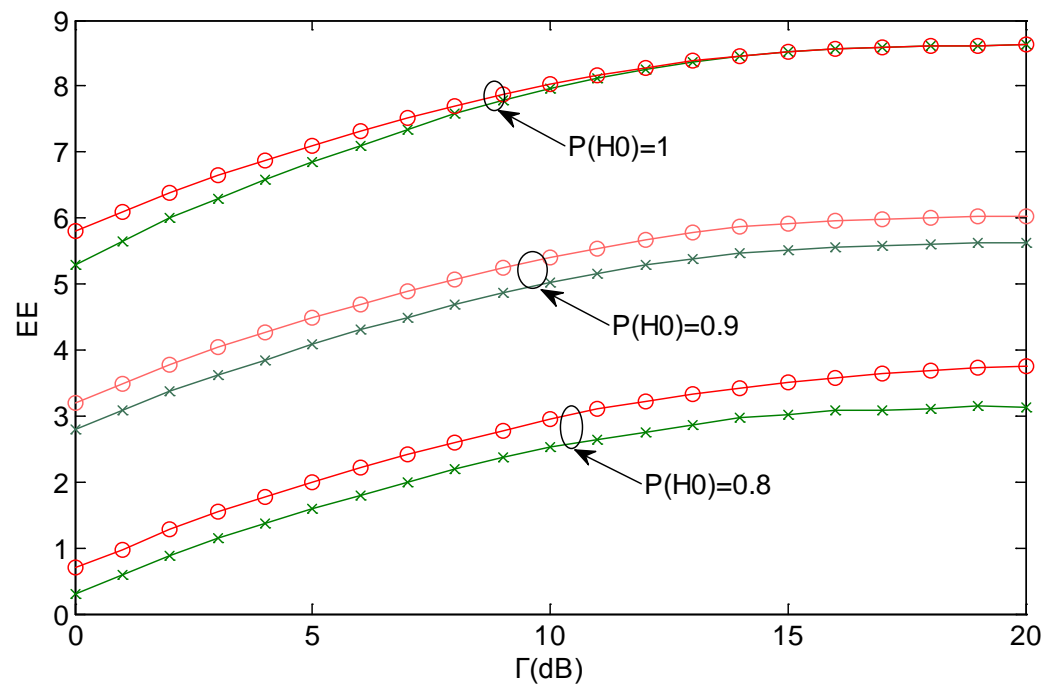

Fig. 5. The EE versus interference power constraint threshold for different probabilities of PU absence.
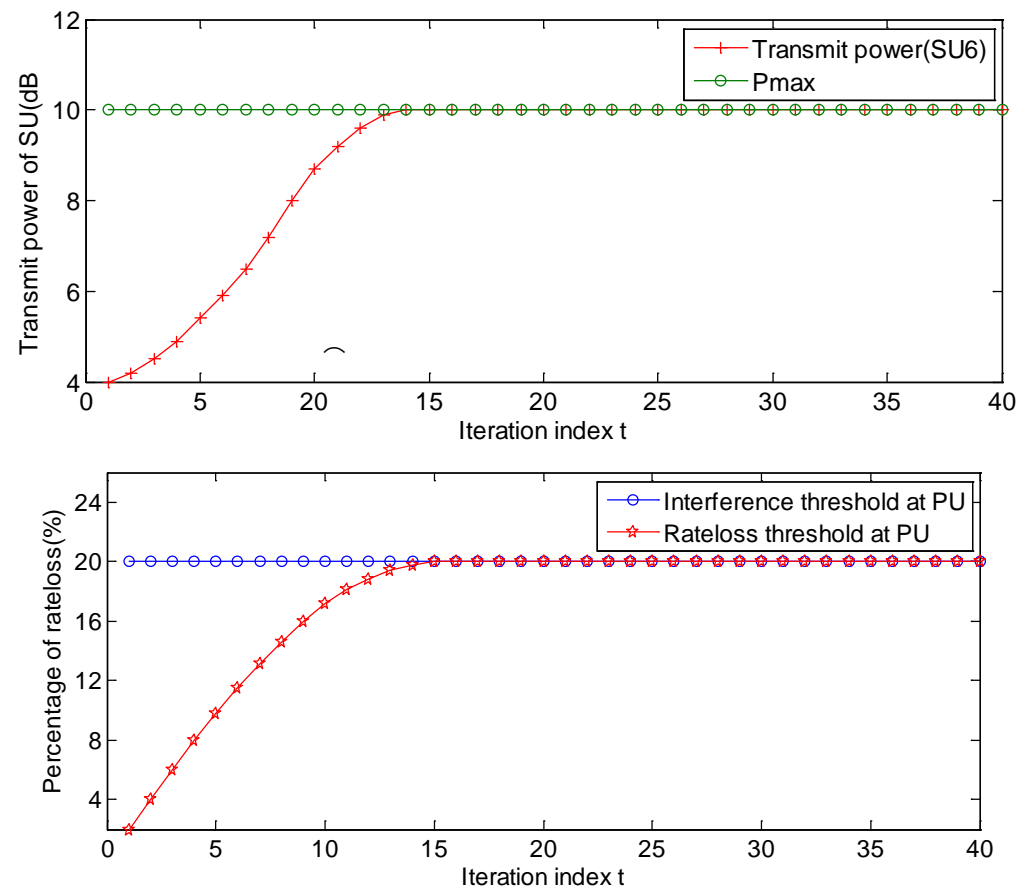

Fig. 6. Convergence performance of the proposed protection.

In Fig. 6, the convergence performance under the proposed constraint have been shown, in terms of the SU's transmission power and the PU's rate loss versus the number of iterations. It is obviously to find that the proposed protection criterion could achieve convergence with small iterations, that indicates a good real-time performance could be achieved for practical applications. As seen from Fig. 6, the proposed protection strategy can well protect the interests of the primary user because the rate loss that the primary femtocell station can tolerate could not be exceeded. 


\section{Conclusion}

The sensing-based rate loss constraint is proposed to be used as a new criterion for SBSS cognitive heterogeneous network in the present work. The resource allocation optimization to maximize energy efficiency under the proposed protection criterion and the conventional interference constraint is designed, respectively. It is proved that under imperfect spectrum sensing situation, the energy efficiency under the new proposed scheme is significantly improved compared with that under the conventional one. The relationship between the proposed protection criterion and the conventional interference constraint has also been investigated. It is proved that the interference power threshold can be regarded as an upper bound of the maximum rate loss that the PU could tolerate. Simulation results have also indicated the effectiveness of the proposed algorithm.

\section{APPENDIX A}

Denote the upper contour sets of $U_{E E}\left(\tau^{*}, P_{s}^{(0)}, P_{s}^{(1)}\right)$ as:

$$
\mathrm{S}_{\rho}=\left\{P_{i, j}^{(s, 0)} \geq 0, P_{i, j}^{(s, 1)} \geq 0 \mid U_{E E}\left(P_{i, j}^{(s, 0)}, P_{i, j}^{(s, 1)}\right) \geq \rho\right\} .
$$

$U_{E E}\left(\tau^{*}, P_{s}^{(0)}, P_{s}^{(1)}\right)$ is strictly quasi-concave respect to $P_{i, j}^{(s, 0)}, P_{i, j}^{(s, 1)}$, respectively, if and only if $\mathrm{S}_{\rho}$ is strictly convex for any real number $\rho$.

When $\rho<0$, no points exist on the contour $U_{E E}\left(\tau^{*}, P_{s}^{(0)}, P_{s}^{(1)}\right)=\rho$. When $\rho=0$, only $P_{i, j}^{(s, 0)}=0$ and $P_{i, j}^{(s, 1)}=0$ is on the contour $U_{E E}\left(\tau^{*}, P_{s}^{(0)}, P_{s}^{(1)}\right)=\rho$. When $\rho<0, \mathrm{~S}_{\rho}$ is equivalent to:

$$
\mathrm{S}_{\rho}=\left\{P_{i, j}^{(s, 0)} \geq 0, P_{i, j}^{(s, 1)} \geq 0 \mid \rho\left(E_{S}\left(\tau, P_{s}^{(0)}, P_{s}^{(1)}\right)+E_{S}(\tau)+E_{C}\right)-\sum_{j=1}^{M} C_{j}\left(\tau, P_{s}^{(0)}, P_{s}^{(1)}\right) \leq 0\right\} .
$$

Proving that $\rho\left(E_{S}\left(\tau, P_{s}^{(0)}, P_{s}^{(1)}\right)+E_{S}(\tau)+E_{C}\right)-\sum_{j=1}^{M} C_{j}\left(\tau, P_{s}^{(0)}, P_{s}^{(1)}\right)$ is strictly convex respect to $P_{i, j}^{(s, 0)}, P_{i, j}^{(s, 1)}$, is easy. Hence, $\mathrm{S}_{\rho}$ is strictly convex and $U_{E E}\left(\tau^{*}, P_{s}^{(0)}, P_{s}^{(1)}\right)$ is strictly quasi-concave respect to $P_{i, j}^{(s, 0)}, P_{i, j}^{(s, 1)}$.

\section{APPENDIX B}

If $\left(P_{i, j}^{(s, 0) *}, P_{i, j}^{(s, 1) *}\right)$ is the optimal solution of (16) associated with the maximum value $q^{*}$, then:

$$
q^{*}=\frac{\sum_{j=1}^{M} C_{j}\left(\hat{\tau}, P_{s}^{(0) *}, P_{s}^{(1) *}\right)}{E_{S}\left(\hat{\tau}, P_{s}^{(0) *}, P_{s}^{(1) *}\right)+E_{S}(\hat{\tau})+E_{C}} .
$$

This equation implies that

$$
\begin{aligned}
& \sum_{j=1}^{M} C_{j}\left(\hat{\tau}, P_{s}^{(0) *}, P_{s}^{(1) *}\right)-q^{*}\left(E_{S}\left(\hat{\tau}, P_{s}^{(0) *}, P_{s}^{(1) *}\right)+E_{S}(\hat{\tau})+E_{C}\right)=0, \\
& \sum_{j=1}^{M} C_{j}\left(\hat{\tau}, P_{s}^{(0) *}, P_{s}^{(1) *}\right)-q^{*}\left(E_{S}\left(\hat{\tau}, P_{s}^{(0) *}, P_{s}^{(1) *}\right)+E_{S}(\hat{\tau})+E_{C}\right) \leq 0 .
\end{aligned}
$$


Then conclude $\mathrm{F}\left(q^{*}, P_{i, j}^{(s, 0), *}, P_{i, j}^{(s, 1), *}\right)=0$.

If $\mathrm{F}\left(q^{*}\right)=\mathrm{F}\left(q^{*}, P_{i, j}^{(s, 0), *}, P_{i, j}^{(s, 1), *}\right)=0$, then $\mathrm{F}\left(q^{*}, P_{i, j}^{(s, 0)}, P_{i, j}^{(s, 1)}\right) \leq \mathrm{F}\left(q^{*}, P_{i, j}^{(s, 0), *}, P_{i, j}^{(s, 1), *}\right)$.

Then:

$$
\frac{\sum_{j=1}^{M} C_{j}\left(\hat{\tau}, P_{s}^{(0) *}, P_{s}^{(1) *}\right)}{E_{S}\left(\hat{\tau}, P_{s}^{(0) *}, P_{s}^{(1) *}\right)+E_{S}(\hat{\tau})+E_{C}}=q^{*} ; \frac{\sum_{j=1}^{M} C_{j}\left(\hat{\tau}, P_{s}^{(0)}, P_{s}^{(1)}\right)}{E_{S}\left(\hat{\tau}, P_{s}^{(0)}, P_{s}^{(1)}\right)+E_{S}(\hat{\tau})+E_{C}} \leq q^{*} .
$$

Thus $\left(P_{k, i}^{\left(0,{ }^{*}, P_{k, i}^{(1) *}\right)}\right.$ is the optimal solution of (3) with the maximum value $q^{*}$.

\section{APPENDIX C}

Proof: when $\kappa_{j}$ is enough large, channel conditions in adjacent subcarriers are often similar. Let $\left(P_{i, j}^{(s, 0, x) *}, P_{i, j}^{(s, 1, x) *}\right)$ and $\left(P_{i, j}^{(s, 0, y)^{*},}, P_{i, j}^{(s, 1, y) *}\right)$ be the optimal transmission power allocation schemes for the energy efficiency optimization problem with rate loss constraints $R_{x, j}$ and $R_{y, j}$. The new power allocation strategy $\left(P_{i, j}^{(s, 0, z)}, P_{i, j}^{(s, 1, z)}\right)$ is constructed in the following way: let power allocation meet $\left(P_{i, j}^{(s, 0, z)}, P_{i, j}^{(s, 1, z)}\right)=\left(P_{i, j}^{(s, 0, x) *}, P_{i, j}^{(s, 1, x) *}\right)$ in the $\chi$ proportion of the subcarriers and $\left(P_{i, j}^{(s, 0, z)}, P_{i, j}^{(s, 1, z)}\right)=\left(P_{i, j}^{(s, 0, y) *}, P_{i, j}^{(s, 1, y) *}\right)$ in the $(1-\chi)$ proportion of the subcarriers. Then a new power allocation strategy in the sensing based rate loss constraint can be achieved as follows:

$$
\begin{array}{r}
R_{j}^{s, z}=\frac{T-\tau}{T} \sum_{i=1}^{K_{j}} \beta_{0, j} \log _{2}\left(1+\frac{g_{p p, i} P_{p, i}}{N_{0}+g_{s p, i} P_{i, j}^{(s, 0, z)}}\right)+\beta_{1, j} \log _{2}\left(1+\frac{g_{p p, i} P_{p, i}}{N_{0}+g_{s p, i} P_{i, j}^{(s, 1, z)}}\right) \\
\quad=\frac{T-\tau}{T} \sum_{i=1}^{K_{j}} \beta_{0, j} \log _{2}\left(1+\frac{g_{p p, i} P_{p, i}}{N_{0}+g_{s p, i} P_{i, j}^{(s, 0, x)}}\right)+\beta_{1, j} \log _{2}\left(1+\frac{g_{p p, i} P_{p, i}}{N_{0}+g_{s p, i} P_{i, j}^{(s, 1, x)}}\right) \\
+\frac{T-\tau}{T} \sum_{i=K_{j}+1}^{K_{j}} \beta_{0, j} \log _{2}\left(1+\frac{g_{p p, i} P_{p, i}}{N_{0}+g_{s p, i} P_{i, j}^{(s, 0, y)}}\right)+\beta_{1, j} \log _{2}\left(1+\frac{g_{p p, i} P_{p, i}}{N_{0}+g_{s p, i} P_{i, j}^{(s, 1, y)}}\right) .
\end{array}
$$

When the number of subcarriers is the law of large number, we have:

$$
R_{j}^{s, z}=\chi R_{j}^{s, \chi}+(1-\chi) R_{j}^{s, y} .
$$

Due to $\left(P_{i, j}^{(s, 0, x) *}, P_{i, j}^{(s, 1, x) *}\right)$ and $\left(P_{i, j}^{(s, 0, y) *}, P_{i, j}^{(s, 1, y) *}\right)$ be the optimal power allocation schemes for the energy efficiency maxization problem, the power allocation schemes satisfy rate loss constraint $R_{x, j}$ and $R_{y, j}$.Then the following conclusion would be have:

$$
\begin{aligned}
& \frac{T-\tau}{T} \sum_{i=1}^{\chi K_{j}} \beta_{0, j} \log _{2}\left(1+\frac{g_{p p, i} P_{p, i}}{N_{0}+g_{s p, i} P_{i, j}^{(s, 0, x)}}\right)+\beta_{1, j} \log _{2}\left(1+\frac{g_{p p, i} P_{p, i}}{N_{0}+g_{s p, i} P_{i, j}^{(s, 1, x)}}\right) \\
& +\frac{T-\tau}{T} \sum_{i=\chi K_{j}+1}^{K_{j}} \beta_{0, j} \log _{2}\left(1+\frac{g_{p p, i} P_{p, i}}{N_{0}+g_{s p, i} P_{i, j}^{(s, 0, y)}}\right)+\beta_{1, j} \log _{2}\left(1+\frac{g_{p p, i} P_{p, i}}{N_{0}+g_{s p, i} P_{i, j}^{(s, 1, y)}}\right) \\
& \geq \chi R_{j}^{x}+(1-\chi) R_{j}^{y} .
\end{aligned}
$$

Therefore, it is clear that $R_{j}^{s, z} \geq \chi R_{j}^{x}+(1-\chi) R_{j}^{y}$. 


\section{APPENDIX D}

Proof: The right hand side function $F\left(P_{i, j}^{(s, 0)}\right)$ and the left hand side function $H\left(P_{i, j}^{(s, 0)}\right)$ in the Theorem 2 is monotonically decreasing respect to the transmission power $P_{i, j}^{(s, 0)}$. For fixed q, $\lambda$ and $\mu_{j}$, the following conclusion should be have.

$$
\begin{gathered}
\lim _{P_{k, i}^{(0)} \rightarrow+\infty} \frac{\alpha_{o, j} g_{s s, i}}{1+g_{s s, i} P_{i, j}^{(s, 0)}}+\frac{\beta_{0, j} g_{s s, i}}{g_{p s, i} P_{p, i}+N_{0}+g_{s s, i} P_{i, j}^{(s, 0)}}=0, \\
\lim _{P_{k, i}^{(0)} \rightarrow+\infty}(q T+\lambda)\left(\alpha_{o, j}+\beta_{0, j}\right)+\frac{\mu_{j} \beta_{0, j} g_{p p, i} g_{s p, i} P_{p, i}}{\left(N_{0}+g_{p p, i} P_{p, i}+g_{s p, i} P_{i, j}^{(s, 0)}\right)\left(N_{0}+g_{s p, i} P_{i, j}^{(s, 0)}\right)}=(q T+\lambda)\left(\alpha_{o, j}+\beta_{0, j}\right) .
\end{gathered}
$$

Therefore, the two function should have the point intersection only if satisfying $F(0) \geq H(0)$. Accordingly, if $F(\phi)=(q T+\lambda)\left(\alpha_{o, j}+\beta_{0, j}\right)$ is satisfied, the intersection point can only be found in the range $[0, \phi]$.

\section{APPENDIX E}

Proof:

$$
\begin{aligned}
R_{j}^{s} & =\frac{T-\tau}{T} \sum_{i \in \mathrm{K}_{j}} \beta_{0, j} \log _{2}\left(1+\frac{g_{p p, i} P_{p, i}}{N_{0}+g_{s p, i} P_{i, j}^{(s, 0)}}\right)+\beta_{1, j} \log _{2}\left(1+\frac{g_{p p, i} P_{i}}{N_{0}+g_{s p, i} P_{i, j}^{(s, 1)}}\right) \\
\geq & \frac{T-\tau}{T}\left\{P\left(H_{1, j}\right)\left(1-p_{d, j}\left(\tau, \varepsilon_{j}\right)\right)\left(\sum_{i \in K_{j}} \log _{2}\left(1+\frac{g_{p p, i} P_{p, i}}{N_{0}}\right)-\sum_{i \in K_{j}} \log _{2}\left(1+\frac{g_{s p, i} P_{i, j}^{(s, 0)}}{N_{0}}\right)\right]\right. \\
& \left.+P\left(H_{1, j}\right) p_{d, j}\left(\tau, \varepsilon_{j}\right)\left[\sum_{i \in K_{j}} \log _{2}\left(1+\frac{g_{p p, i} P_{p, i}}{N_{0}}\right)-\sum_{i \in K_{j}} \log _{2}\left(1+\frac{g_{s p, i} P_{i, j}^{(s, 1)}}{N_{0}}\right)\right]\right\} \\
= & \frac{T-\tau}{T} \sum_{i \in \mathrm{K}_{j}} P\left(H_{1, j}\right) \log _{2}\left(1+\frac{g_{p p, i} P_{p, i}}{N_{0}}\right)-P\left(H_{1, j}\right)\left(1-p_{d, j}\left(\tau, \varepsilon_{j}\right)\right) \log _{2}\left(1+\frac{g_{s p, i} P_{i, j}^{(s, 0)}}{N_{0}}\right) \\
- & P\left(H_{1, j}\right) p_{d, j}\left(\tau, \varepsilon_{j}\right) \log _{2}\left(1+\frac{g_{s p, i} P_{i, j}^{(s, 1)}}{N_{0}}\right) \\
\geq & \frac{T-\tau}{T}\left\{P\left(H_{1, j}\right) \sum_{i \in K_{j}} \log _{2}\left(1+\frac{g_{p p, i} P_{p, i}}{N_{0}}\right)\right. \\
& \left.-P\left(H_{1, j}\right)\left(1-p_{d, j}\left(\tau, \varepsilon_{j}\right)\right) \sum_{i \in K_{j}} \frac{g_{s p, i} P_{i, j}^{(s, 0)}}{N_{0} \ln 2}-P\left(H_{1, j}\right) p_{d, j}\left(\tau, \varepsilon_{j}\right) \sum_{i \in K_{j}} \frac{g_{s p, i} P_{i, j}^{(s, 1)}}{N_{0} \ln 2}\right\} \\
\geq & R_{j}^{p}-\frac{\mathrm{K}_{j}}{N_{0} \ln 2} \Gamma_{i, j} \cdot
\end{aligned}
$$

\section{References}

[1] J. Mitola III and G. Q. Maguire, Jr., "Cognitive radios: making software radio more personal," IEEE Personal Commun., vol. 6, no. 4, pp. 13-18, Aug. 1999. Article (CrossRef Link) 
[2] Li Xu, He Fang, Zhiwei Lin, "Evolutionarily stable opportunistic spectrum access in cognitive radio networks,” IET Communications, vol. 10, no. 17, pp.2290-2299, Nov. 2016. Article (CrossRef Link)

[3] Lokman Sboui, Hakim Ghazzai, Zouheir Rezki, "Achievable Rate of Spectrum Sharing Cognitive Radio Multiple-Antenna Channels,” IEEE Transactions on Wireless Communications, vol. 14, no. 9, pp.4847-4856, Sep. 2015. Article (CrossRef Link)

[4] Shuying Zhang, Xiaohui Zhao, "Power allocation for sensing-based spectrum sharing cognitive radio system with primary quantized side information,” China Communications, vol. 13, no. 9, pp. 33-43, Sep. 2016. Article (CrossRef Link)

[5] Van-Dinh Nguyen, Oh-Soon Shin, "Cooperative Prediction-and-Sensing-Based Spectrum Sharing in Cognitive Radio Networks,"IEEE Transactions on Cognitive Communications and Networking, vol. 4, no. 1, pp. 108-120, March. 2018. Article (CrossRef Link)

[6] X. Kang, Y-C. Liang, A. Nallanathan, H. K. Garg, R. Zhang, "Optimal power allocation for fading channels in cognitive radio networks: ergodic capacity and outage capacity,” IEEE Transactions on Wireless Communications, vol. 8, no. 2, pp. 940-950, Feb. 2009.

Article (CrossRef Link)

[7] Wenjie Zhang, Yingjuan Sun, Lei Deng, Chai Kiat Yeo, and Liwei Yang, "Dynamic Spectrum Allocation for Heterogeneous Cognitive Radio Networks With Multiple Channels,” IEEE System Journal, vol. 9, no.6, pp. 1-12, April 2018. Article (CrossRef Link)

[8] A.Zaeemzadeh, M. Joneidi, N. Rahnavard, GJun Qi, “Co-SpOT: Cooperative Spectrum Opportunity Detection using Bayesian Clustering in Spectrum-Heterogeneous Cognitive Radio Networks," IEEE Transactions on Cognitive Communications and Networking, vol. 4, no. 2, pp. 206-219, June 2018. Article (CrossRef Link)

[9] Zengmao Chen, Yunmin Tang, Zhiguo Sun, “An interference model for heterogeneous cognitive radio networks,” 2017 IEEE/CIC International Conference on Communications in China (ICCC), Jan. 2017. Article (CrossRef Link)

[10] M. Munochiveyi, Xiaohui Zhao, J. Mudare, "Wigner-Ville Distribution MVDR Beamforming Scheme for Interference-Tolerant Cognitive Radio Network,” 2017 IEEE/CIC International Conference on Communications in China (ICCC), Jan. 2017. Article (CrossRef Link)

[11] Woping Xu, Runhe Qiu, Julian Cheng, "Fair Optimal Resource Allocation in Cognitive Radio Networks With Co-Channel Interference Mitigation,” IEEE Access, vol. 2, no. 1, pp. 37418 37429, Feb. 2018. Article (CrossRef Link)

[12] M.Jo, T. Maksymyuk, L. Batista, F.Maciel, F.de Almeida, M.Klymash, “A Survey of Converging Solutions for Heterogeneous Mobile Networks,” IEEE Wireless Communications, Vol.21, No.8, pp.54-62, Dec. 2014. Article (CrossRef Link)

[13] X. Kang, Y.-C. Liang, H. K. Garg, R. Zhang, "optimal power allocation for OFDM-based cognitive radio with new primary transmission protect criteria," IEEE Transactions on Wireless Communications, vol. 9, no.6, pp. 1536-1276, June. 2010. Article (CrossRef Link)

[14] D. Li, J. Cheng, V. Leung, “Adaptive Spectrum Sharing for Half-duplex and Full-duplex Cognitive Radios: From the Energy Efficiency Perspective," IEEE Transactions on Communications, vol. 9, no.6, pp. 1-12, June. 2018. Article (CrossRef Link)

[15] X. Zhai, K. Chen, X. Liu, "Energy Efficiency of Access Control with Rate Constraints in Cognitive Radio Networks,” IEEE Access, vol. 6, pp.36354 - 36363, Sep. 2018. Article (CrossRef Link)

[16] X. Liu, K. Zheng, L. Fu, X. Liu, X. Wang, G. Dai, "Energy Efficiency of Secure Cognitive Radio Networks with Cooperative Spectrum Sharing," IEEE Transactions on Mobile Computing, vol. 6, pp.1536 -1233, May, 2018. Article (CrossRef Link)

[17] M. Zhou, H. Yin, H. Wang, "Robust Energy Efficiency Power Allocation Algorithm for Cognitive Radio Networks with Rate Constraints,” in Proc. of 2017 17th IEEE International Conference on Communication Technology, pp.849 -854, 2017. Article (CrossRef Link)

[18] Xu yongjun, Hu yuan, Li guoquan, Zhang haibo. "Robust resource allocation for heterogeneous wireless networks: a worst-case optimization,” IETCommunications, vol.12, no. 9, pp. 1064-1071, June 2018. Article (CrossRef Link) 
[19] S.Stotas, A.Nallanathan, "Optimal sensing time and power allocation in multiband cognitive radio network,” IEEE Transactions on Communications, vol. 59, no.1, pp.226 - 235, Jan. 2018. Article (CrossRef Link)

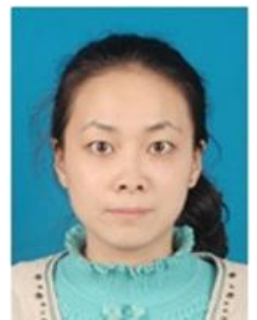

Bing Ning received the Ph.D. degree in information and communication system from Zhengzhou University, Zhengzhou, China, in 2016 and the B.E. degree in Electronic Information Engineering from the Nanjing Artillery Academy of PLA, Nanjing, China, in 2009. She is currently working in Zhongyuan university of Technology as a lecturer. From October 2012 to January 2013, she was a visiting researcher with the Department of Electrical Engineering and Computer Science, University of Kansas, Lawrence, USA. Her research interests are in the areas of wireless communications, including cognitive radio, Non-Orthogonal Multiple Access, and Multiple-input multiple-output.

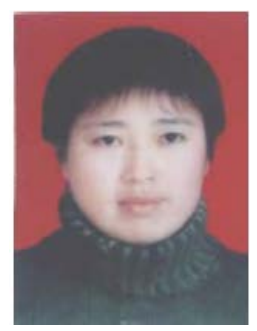

Aihua Zhang received the B.S. and Ph.D. degrees from Zhengzhou University of china in 1998 and 2014, respectively. She received the master degree from China University of Mining \\& Technology-Beijing in 2003. From 2003 to 2014, she was a Lecturer. Since 2014, she has been an Associate Professor with Zhongyuan University of Technology. Her current interests are in the areas of signal processing in communication system, wireless communications.

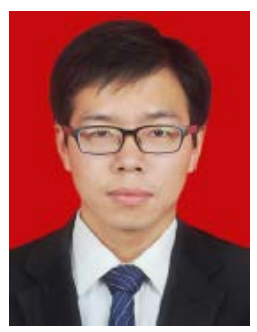

Wanming Hao received the Master degree from Zhengzhou University, China in 2015. Now, He is currently serving as a Ph.D.Student at Kyushu University, Japan. His research interests include broadband wireless communication, cognitive radio, cooperative communication, Massive MIMO.

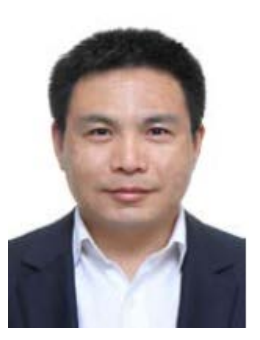

Jianjun Li received the B.S. and the M.S. degree from Xidian University, Xi'an, China, and the Ph.D. degree in electronic engineering from Tsinghua University, Beijing, China in 1996, 1999 and 2002, respectively. From 2002 2007, he was a senior researcher on 4G wireless communication in Beijing Samsung Telecom. R।\&D Center in Beijing China. From 2007 2015, he has worked in Posdata, Pantech and Innovative technology Lab. Co. as a senior researcher in Korea. During these period, his research mainly focus on Wimax and 3GPP LTE standard including MIMO, CoMP, small cell and LAA. Since 2016, he has been a Professor at Zhongyuan University of Technology. His current research interest is on 5G wireless communication including massive MIMO, NOMA, new waveform and application of digital signal processing in communication systems.

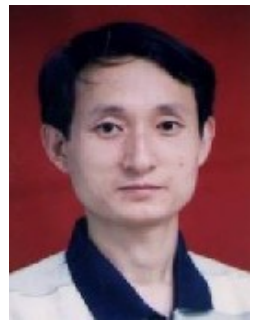

Shouyi Yang received his Ph.D degree from Beijing Institute of Technology, China in 2002. He is currently a Full Professor with the School of Information Engineering, Zhengzhou University, China. He has published various paper in the field of signal processing and wireless communication. His research interests include signal processing in communications system, wireless communications and cognitive radio. 